\title{
Visual Acuity and Contrast Sensitivity in Cataract: Summation and Inhibition of Visual Performance
}

\author{
ROBERT H. TAYLOR ${ }^{1}$, GARY P. MISSON ${ }^{1}$, MERRICK J. MOSELEY ${ }^{2}$ \\ Birmingham
}

\begin{abstract}
Summary
Patients exhibiting uniocular cataract often report an improvement in vision on closing their cataractous eye. Such qualitative evidence suggests the presence of binocular inhibition - the converse of binocular summation (that is the normal superiority of binocular over monocular vision). To quantify the extent of inhibition in cataract, binocular and monocular visual acuity and contrast sensitivity were measured in 28 patients. Twelve patients showed binocular inhibition for visual acuity whilst 11 showed inhibition for contrast sensitivity measured at four cycles per

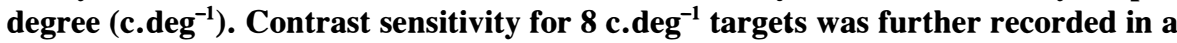
subset of 14 patients in whom seven showed inhibition. In patients who demonstrated inhibition, the mean decrement in performance for visual acuity was $13 \%$. For contrast sensitivity at 4 and $8{\mathrm{c} . \mathrm{deg}^{-1}}^{-1}$ the mean decrement was $25 \%$ and $32 \%$ respectively. The clinical significance of these findings is discussed with respect to the assessment of visual function and management of the cataract patient.
\end{abstract}

Vision with two eyes is generally superior to that with a single eye. The improvement in performance is termed binocular summation and is independent of any benefit attributable to stereopsis.

Binocular summation has been studied empirically since Jurin's early observations. ${ }^{*}$ It occurs predominantly with simple visual tasks and in most cases the magnitude of the effect exceeds that predicted on the basis of probability summation. ${ }^{2,3}$

If the vision of one eye is artificially reduced during binocular viewing (e.g. by blurring lenses, glare or a reduction in retinal illuminance) visual performance may fall below that of monocular vision. ${ }^{4-6}$ This paradoxical con- verse of summation has been termed binocular inhibition.?

In the clinical domain, the presence of a uniocular cataract can be considered analogous to the conditions under which binocular inhibition has been observed in the laboratory, indeed patients often allude to an improvement of sight on closing their cataractous eye. A recent laboratory investigation reported by Pardhan and Gilchrist ${ }^{7}$ has confirmed the presence of binocular inhibition of contrast sensitivity in uniocular cataract. These authors observed the magnitude of inhibition to be related to the severity of the visual impairment and the spatial frequency of the test target.

\footnotetext{
*Jurin (1738) cited in Jones and Lee. ${ }^{1}$

${ }^{1}$ The Birmingham and Midland Eye Hospital, Church Street, Birmingham

${ }^{2}$ Department of Ophthalmology, University of Birmingham

Correspondence to: Dr Merrick J Moseley, Lecturer, Academic Unit, Birmingham and Midland Eye Hospital, Church Street, Birmingham B3 2NS.
} 
Given the laboratory and anecdotal evidence that patients with unequal vision due to cataract may suffer an inhibition of binocular vision, the question arises as to whether the phenomenon may be usefully measured for clinical purposes. In this paper we compare the binocular and monocular visual performance of a group of cataract patients to determine the extent and magnitude of binocular inhibition as revealed by standard tests of spatial vision.

\section{Methods}

Twenty-eight patients (age range: 35-84 years, mean: 64.7 years) were recruited from the pre-operative ward round at The Birmingham and Midland Eye Hospital. Subjects were provided with a full explanation of the purpose of the study and their verbal consent obtained. Prior ethical approval had been granted by the District Ethical Committee, West Birmingham Health Authority. All subjects had cataract in one eye which considerably reduced the vision compared to the other, 'good' eye. In 14 cases previous cataract surgery had been performed.

Monocular and binocular recognition visual acuities were recorded using a BaileyLovie $\log$ MAR chart ${ }^{8}$. The advantages of using $\log$ MAR charts to record visual acuity have been described elsewhere ${ }^{9}$ but briefly, they include the ability accurately to record acuities where only part of a line is read, a scale that closely approximates to human visual discriminability, and the control of contour interaction. Acuities were scored according to the interpolation method of Kitchin and Bailey ${ }^{10}$ whereby each letter read correctly was deemed to increase the acuity score by $0.02 \log$ MAR (one fifth of a line). The standard test distance was six metres. If subjects were unable to read the largest optotypes (1.0 $\log$ MAR, 6/60), viewing distances were reduced as appropriate.

Contrast sensitivity was measured using the Cambridge Low Contrast Gratings ${ }^{11}$ at a test distance of six metres (corresponding to a spatial frequency of four cycles per degree). In a subset of 14 subjects the test was further per-

$\dagger$ Test results were converted to contrast sensitivity scores (1/Michelson contrast) using tables supplied with the Cambridge Low Contrast Gratings. formed at a distance of 12 metres (corresponding to a spatial frequency of eight cycles per degree). Both acuity and contrast sensitivity were measured for each eye in isolation and with both eyes open. Normal distance spectacle correction was worn at all times.

On completion of the assessment of acuity and contrast sensitivity, slit-lamp examinations were performed after instillation of a mydriatic agent (Tropicamide 1\%). Cataract was evaluated in three regions: anterior capsular, nuclear and/or posterior subcapsular and a grading of zero to three was made for each of these areas. The amount of brunescence and white scatter was assessed according to the Oxford Clinical Cataract Classification and Grading System ${ }^{12}$.

\section{Results}

Ranges of visual acuity for both eyes, the non-cataractous eye and the cataractous eye were respectively -0.02 to $0.74,0.04$ to 0.76 and 0.44 to $1.78 \log$ MAR. Corresponding measures of contrast sensitivity $\dagger$ were 16 to 480,10 to 440 and 10 to 88 for the $4{\mathrm{c} . \mathrm{deg}^{-1}}^{-1}$ gratings and 10 to 190,10 to 340 , and 10 to 16 for the $8 \mathrm{c} \cdot \mathrm{deg}^{-1}$ gratings.

For visual acuity, 12 subjects demonstrated binocular summation, 12 inhibition and 4 neither inhibition nor summation. For con-

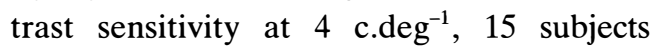
demonstrated summation, 11 inhibition and two neither inhibition nor summation. At

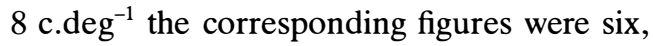
seven and one.

Comparisons between viewing conditions were performed using the non-parametric Wilcoxon Sign Ranks Test. As expected, acuity and contrast sensitivity in the cataractous eye were significantly poorer $(p<0.001)$ than in either the 'good' eye or with binocular viewing. Comparisons between binocular visual performance and monocular performance of the non-cataractous eye revealed no significant difference in visual acuity or contrast sensitivity at

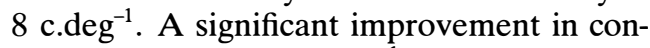
trast sensitivity at $4 \mathrm{c} . \mathrm{deg}^{-1}$ occurred for binocular over monocular (non-cataractous eye, $\mathrm{p} \leqslant 0.001$ ) viewing.

\section{Discussion}

To evaluate the binocular inhibition found in 
our patient sample it is pertinent to consider the magnitude of summation present in normal eyes. Recent data obtained by Heravian et al. ${ }^{13}$ found the mean binocular summation for Landolt ' $\mathrm{C}$ ' targets to be $11.3 \%$ (range 1 to $17 \%$ ). For contrast sensitivity, a figure of $41 \%$ has been obtained by Campbell and Green ${ }^{14}$. Given these figures relating to the normal population, it is significant that over one-third of the patients tested in our study demonstrated poorer binocular visual performance (acuity and contrast sensitivity) than when monocular viewing with the 'good' eye. For acuity, of those patients showing inhibition, the reduction in performance averaged $13 \%$ (range 4 to $43 \%$ ). This corresponds to a mean decrement of approximately half a line. For contrast sensitivity at $4 \mathrm{c} . \mathrm{deg}^{-1}$ the mean percentage inhibition was $25 \%$ (range 7 to $52 \%$ ) and at $8 \mathrm{c} \cdot \mathrm{deg}^{-1}, 32 \%$ (range 10 to $70 \%)$.

What factors determine whether cataract patients are likely to suffer binocular inhibition? Neither catagory, grade nor visual acuity of the cataractous eye was able to predict the presence or magnitude of inhibition so as yet this question must remain unanswered. It has been argued $^{7}$ that extremely poor vision within the cataractous eye acts as an occluder thus eliminating inhibitory effects, however this was not apparent in our data-one subject with a monocular acuity in the cataractous eye of $1.78 \log$ MAR (worse than $6 / 360$ ) was found to have a monocular acuity of the non-cataractous eye more than two lines better than with binocular viewing.

The management of patients with cataract requires an asssessment of their visual disability. The best corrected Snellen visual acuity is usually recorded and is considered together with each patient's requirements as dictated by their life style. Having taken these factors into account, the timing of surgery is largely based on the acuity of the cataractous eye. The acuity of the 'good' eye is also considered, if only to assess the degree of urgency. We have shown that the measurement of monocular visual acuity is not always a representation of true visual function. Over one-third of our subjects demonstrated binocular inhibition of visual acuity, and contrast sensitivity at $4 \mathrm{c} \cdot \mathrm{deg}^{-1}$. At $8 \mathrm{c} \cdot \mathrm{deg}^{-1}$ the pro- portion was $50 \%$. These data suggest that binocular inhibition is common in uniocular cataract. Interestingly only three of the 12 subjects exhibiting inhibition as measured by acuity also exhibited inhibition as measured by contrast sensitivity at $4 \mathrm{c} \cdot \mathrm{deg}^{-1}$. The reason for this is unclear but it suggests that measurement of both acuity and contrast sensitivity may be necessary to test for the presence of inhibition. However the magnitude of inhibition demonstrated with contrast sensitivity was approximately twice that shown for visual acuity. Also worthy of note is the reduced likelihood of detecting the typically small amounts of inhibition with a standard Snellen chart due to its unequal scale increments and number of characters per line.

Contrast sensitivity tests in a form suitable for clinical use are becoming increasingly available (e.g. The Arden Grating Test, The Pelli-Robson Chart, The Cambridge Low Contrast Gratings). Our findings suggest that such rapid, hard-copy tests of contrast sensitivity may be a useful adjunct to conventional acuity assessment in cataract. Further investigation is required to identify factors which may predict the likelihood of binocular inhibition.

Our findings give credence to the patient who complains that they are able to manage better with the poorer eye occluded. The presence of one eye with good vision should not deter the clinician from considering early surgery in the other eye.

The authors wish to thank Shahina Pardhan for he, helpful correspondence, and Alistair Fielder and Philip Murray for their thoughtful advice.

Key words: binocular summation, binocular inhibition, cataract, contrast sensitivity, visual acuity.

\footnotetext{
Referenees

${ }^{1}$ Jones RK and Lee DN: Why two eyes are better than one: the two views of binocular vision. J Exp Psychol 1981, 7: 30-40.

${ }^{2}$ Blake R and Fox R: The psychophysical inquiry inte binocular summation. Percept Psychophys 1973. 14: 431-6.

${ }^{3}$ Frisén L and Lindblom B: Binocular summation in humans: evidence for a hierarchic model. $J$ Physiol 1988, 402: 773-82.

${ }^{4}$ Gilchrist $\mathbf{J}$ and Pardhan S: Binocular contrast detection with unequal monocular illuminance. $\mathrm{Oph}$. thal Physiol Opt 1987, 7: 373-7.
} 
${ }^{5}$ Pardhan S and Gilchrist J: The effect of monocular defocus on binocular contrast sensitivity. Ophthal Physiol Opt 1990, 10: 33-5.

${ }^{6}$ Pardhan S and Gilchrist J: Binocular contrast sensitivity with monocular glare disability. Ophthal Physiol Opt 1990, 10: 37-9.

${ }^{7}$ Pardhan $\mathrm{S}$ and Gilchrist $\mathrm{J}$ : The importance of measuring binocular contrast sensitivity in unilateral cataract. Eye 1991, 5: 31-5.

${ }^{8}$ Bailey IL and Lovie JE: New design principles for visual acuity letter charts. Am J Optom Physiol Opt 1976, 53: 745-53.

${ }^{9}$ Rubin GS: Assessment of visual function in eyes with visual loss. In Fuller DG and Birch DG eds. Assessment of visual function for the clinician: Ophthalmology Clinics of North America: 1989, 2(3): 357-67.

${ }^{10}$ Kitchin JE and Bailey IL: Task complexity and visual acuity in senile macular degeneration. Aust $J$ Optom 1981, 64: 235-42.

${ }^{11}$ Wilkins AJ, Della Sala S, Somazzi L, Nimmo-Smith I: Age-related norms for the Cambridge Low Contrast Gratings including details concerning their design and use. Clin Vision Sci 1987, 2: 210-12.

${ }^{12}$ Sparrow JM, Bron AJ, Brown NAP, Ayliffe W, Hill AR: The Oxford Clinical Cataract Classification and Grading System. Int Ophthalmol 1986, 9: 207-25.

${ }^{13}$ Heravian JS, Jenkins TCA, Douthwaite WA: Binocular summation in visually evoked responses and visual acuity. Ophthal Physiol Opt 1990, 10: 257-61.

${ }^{14}$ Campbell F and Green D: Monocular versus binocular visual acuity. Nature (Lond) 1965, 208: 191-2. 